\title{
Le Forum pour les Femmes Actives: Lutte pour le crédit et le changement
}

Marty Chen

Follow this and additional works at: https://knowledgecommons.popcouncil.org/departments_sbsr-pgy

Part of the Family, Life Course, and Society Commons, Gender and Sexuality Commons, Inequality and Stratification Commons, International Public Health Commons, and the Regional Economics Commons How does access to this work benefit you? Let us know!

\section{Recommended Citation}

Chen, Marty. 1983. "Le Forum pour les Femmes Actives: Lutte pour le crédit et le changement," SEEDS no. 6. New York: Population Council. 
SEEDS est une série de brochures publiées en réponse aux demandes d'information venant du monde entier sur des idées de programmes innovatrices et pratiques, réalisées par et pour des femmes aux revenus faibles. Le but de ces brochures est de diffuser l'information et de stimuler la création de nouveaux projets, fondés sur les expériences positives de femmes qui travaillent pour améliorer leur propre statut économique et celui des autres. Les projets décrits dans ce numéro comme dans le reste de la série ont été choisis du fait qu'ils procurent aux femmes un revenu en espèces et les font participer activement aux prises de décisions comme aux profits. Ces projets sont bâtis suivant des critères économiquees solides et permettent de surmonter avec succès des obstacles courants. Ces rapports n'ont cependant pas été conçus pour être suivis à la lettre car chaque effort de développement doit faire face à des ressources et des problèmes quelque peu différents. En revanche, ils relatent l'histoire d'une idée et sa mise en application avec l'espoir que les leçons apprises se révéleront utiles dans des environnements variés. C'est également pour être portés à l'attention de ceux qui détiennent les pouvoirs de décision qu'ils ont été rédigés et leur montrer que des projets générateurs de revenus, pour et par des femmes, sont viables et jouent un rôle important dans le développement.

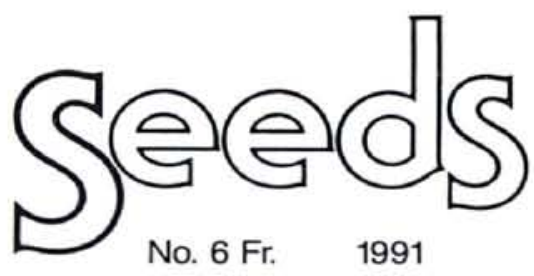

ISSN 073-6833

Copyright (c) 1991 SEEDS
La publication de SEEDS est administrée par le Population Council. Les critères de publication sont déterminés par le Comité de Rédaction de SEEDS: Judith Bruce (The Population Council), Marty Chen (Harvard Institute for International Development), Margaret Clark (The Aspen Institute), Anne Kubisch (The Ford Foundation), Ann

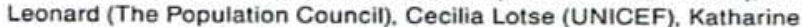
McKee (Center for Community Self-Help), Anne Walker (International Women's Tribune Center) et Mildred Warner (Cornell University).

Les opinions et commentaires exprimès dans cette brochure sont la seule responsabilité de l'auteur et n'engagent que lui. 


\title{
Le Forum pour les Fermmes Actives: Lutte pour le Crédit et le Changement
}

\author{
par Marty Chen
}

\section{Introduction}

La plupart des femmes demeurant dans les bidonvilles de Madras, une large ville au sud de l'Inde, s'adonnent à la vente pour subvenir aux besoins de leur famille. Pourtant, les hommes politiques et les planificateurs ne tiennent pas compte de ces activités économiques qui sont perçues comme étant simplement un supplément aux revenus des hommes et, par conséquent, insignifiantes. En réalité, la contribution des ces femmes entrepreneurs s'élève à 50\% du revenu annuel de la famille.

Pendant des années, ces femmes ont poursuivi leurs activités en passant littéralement inaperçues aux yeux de la société. C'est alors qu'en 1978, un groupe de femmes, politiquement et socialement actives, déçues par les maigres résultats obtenus par les approches traditionnelles mises en oeuvre pour venir en aide aux femmes pauvres, décidèrent de remédier à cette situation. En parlant directement aux femmes vivant dans les bidonvilles, elles ont réalisées que la plupart d'entre elles travaillaient pour leur propre compte et que leur souci principal était non pas comment résoudre leurs problèmes sociaux mais comment obtenir du crédit pour pouvoir maintenir et élargir leur affaire.

C'est pour répondre à ce besoin que fut crée le Forum pour les Femmes Actives. Ce numéro retrace les différentes étapes qui ont mené à la création du Forum et comment plus de 13,000 femmes pauvres ont pu, grâce à cette organisation, avoir accès au crédit. II décrit également comment le Forum a diversifié ses activités pour fournir à ses membres d'autres services, notamment dans le domaine de l'éducation, la santé et la planification familiale et montre comment le fait de se sentir solidaires et reconnues permet à ses membres de mieux faire face aux problèmes politiques et sociaux qui affectent leur vie. 


\section{Les Débuts}

Surnommée "métropole rurale", Madras, la capitale d'un large état au sud de l'Inde, est la quatrième ville la plus grande de l'Inde avec une population de 2,3 millions d'habitants. En grandissant, la ville a englouti les hameaux environnants, ce qui fait que la plupart des bidonvilles sont en fait des villages constitués de cabanes plutôt que des maisons à plusieurs étages. Pourtant, l'économie dans ces banlieues n'est plus une économie rurale. Les habitants, des travailleurs journaliers ou des petits commerçants, font de leur mieux afin de pourvoir aux besoins en biens et en services de la métropole.

La plupart des hommes demeurant dans les bidonvilles n'ont pas de poste fixe mais font du travail temporaire trois ou quatre jours par semaine. Les femmes, quant à elles, s'adonnent à ce que l'on appelle des "autres services" tels que le petit commerce ou le colportage. Elles travaillent généralement tous les jours de la semaine et gagnent, en moyenne, l'équivalent de USD 20 par mois, ce qui représente environ la moitié du revenu total de la famille. $\mathrm{Et}$, compte tenu de l'irrégularité des salaires des hommes vu qu'ils n'ont pas de poste fixe et de leurs habitudes de consommation (par exemple, dépenses fréquentes pour le tabac et l'alcool), on se rend compte que les familles vivant dans les bidonvilles dépendent principalement pour leur survie du revenu des femmes. Par ailleurs, une femme sur dix est le seul soutien financier de la famille.

En novembre 1977, Jaya Arunachalam, une assistante sociale et militante politique très connue, s'occupait de venir en aide aux victimes d'innondations à Madras. Lorsqu'elle se rendit dans les régions sinistrées pour parler aux femmes, elle eu la surprise de découvrir que ces dernières ne s'intéressaient nullement au plan de secours ni à la politique. Leur préoccupation première était de pouvoir gagner de l'argent, et l'obstacle majeur qu'elles rencontraient était le manque de crédit. Voici ce que nous raconte une des collègues de Jaya, qui est, à présent, Vice-Présidente du Forum pour Les Femmes Actives:

Entre Octobre 1977 et Février 1978, Jaya et moi sommes allées en mission dans les banlieues de Madras. Nous nous sommes rendues compte que nous avions tort de convier les femmes à des rassemblements politiques... Nous n'avions rien à leur offrir. En parlant avec elles en petits groupes, nous avons découvert que chaque femme avait une petite affaire et avait contracté des dettes auprès de prêteurs locaux. C'est alors que nous avons pris la décision de rallier les femmes autour de questions économiques.

Une excellente occasion se présenta bientôt pour Jaya. Elle devait prononcer un discours devant des hauts-fonctionnaires de la Banque d'Inde et elle en profita pour leur lancer un défi en posant la question suivante: "Pourquoi ne faites vous rien pour les femmes pauvres?" En Inde, depuis leur nationalisation en 1969, les banques avaient, entre autre, pour mission d'accorder des prêts aux pauvres. Pourtant, aucun effort n'avait été déployé pour informer les femmes pauvres qu'il leur était possible d'obtenir du crédit. Le gérant de la banque locale, un homme exceptionnellement libéral et ouvert, releva le défi et accepta de rencontrer et de financer des groupes de femmes économiquement faibles si ces groupes s'avéraient solvables.

Avec l'appui de Jaya, 30 femmes ont formé un groupe, rencontré le gérant et reçu chacune un prêt de 300 roupies (USD 33). Le groupe a ensuite élu une responsable chargée de collecter l'argent chaque jour auprès des membres du groupe en vue du remboursement du prêt à la Banque d'Inde. Quelques mois suffirent pour démontrer que le système marchait. Le taux de remboursement était de 95\%. En avril 1978, 800 femmes réparties en 40 groupes avaient obtenu des prêts. Le Forum pour les Femmes Actives était né.

Bien que l'accès au crédit était la raison pour laquelle le Forum avait été crée, ses responsables réalisèrent très rapidement que les perspectives économiques des femmes étaient limitées en raison du contexte politique et social. C'est la raison pour laquelle elles ont décidé de poursuivre les objectifs suivants:

- créer une association pour les femmes travaillant pour leur propre compte et n'appartenant à aucune organisation professionnelle.

- identifier et pourvoir aux besoins les plus cruciaux des femmes actives. 

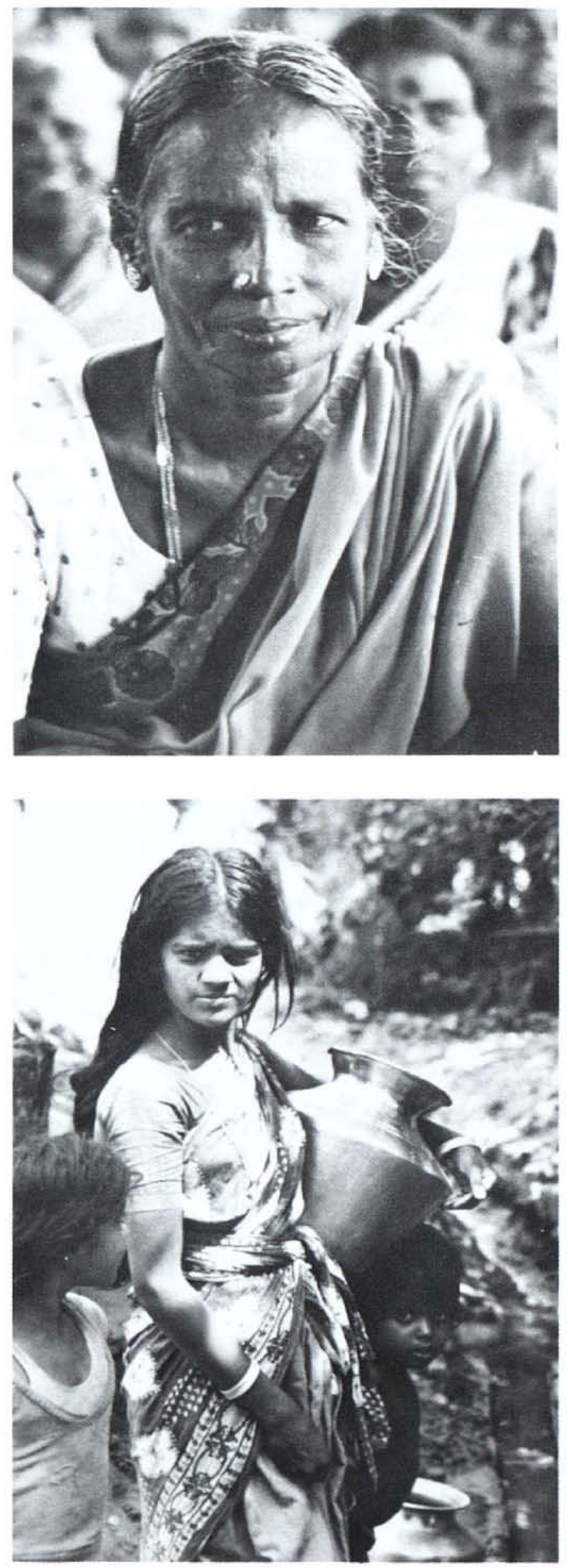

- mobiliser les femmes actives pour constituer un groupe de pression dans le but de revendiquer leurs droits politiques et sociaux.

- aider les femmes actives à mieux gérer leurs affaires par des stages de formation, le crédit et la formation continue, et

- mettre en place des services de soutien indispensables aux femmes actives et leur famille (tels que la garde des enfants, l'éducation, la santé et la planification familiale).

Parallèlement, pour éviter que des différences de caste, de religion ou de politique ne sèment la discorde parmi ses membres, les fondatrices du Forum ont adopté certaines positions idéologiques fermes. Le Forum allait être:

- Pro-femmes: mobilisation exclusive des femmes qui sont le pivot de la famille aussi bien en termes de revenu que de bienêtre.

- Anti-caste et Pro-laïque: favoriser et encourager l'échange entre les femmes appartenant à différentes castes ou groupements religieux, les mariages intercastes et la tolérance religieuse.

- Anti-politique: éviter de prendre un engagement politique quelconque mais sensibiliser les femmes sur des questions touchant aux femmes et aux pauvres.

- Anti-dot: organiser des manifestations de masse pour dénoncer les dots, le viol et le divorce.

\section{Les Femmes}

Selon un recensement mené en 1971, au moins 23,000 femmes vivant dans les bidonvilles de Madras travaillaient dans la catégorie "autres services". Pourtant, ce ne fut qu'après la création du FFA que l'on commença à avoir une idée plus précise des activités économiques de ces femmes. Le Forum identifia plus de 65 petits commerces et affaires tenus par ses membres que l'on pouvait répartir dans les quatre catégories suivantes: vente de légumes, 'stands "pour passer le temps" (vente de denrées alimentaires), vente de coupons de tissus, et vente de fleurs et de fruits. 


\section{Commerces Tenus par les Membres du Forum}

(répertoriés d'après les demandes de prêts)

Vente de Légumes

Marchande de Sari et de Coupons de Tissus

Marchande de Fruits

Fripière

Marchande de Légumes Verts

Vente d'Habits de Confection

Marchande de Poissons

Marchande de Bois de Chauffage

Marchande d'Ustensiles en Aluminium Epicière

Fabricante d'Encens

Marchande de Soieries

Fabricante de Pandal (ornements)

Fabricante de Fleurs en Plastiques

Propriétaire de Stand de Thé

Propriétaire d'un Stand de Poteries

Marchande de Foin

Propriétaire de Snack-Bar

Fabricante de Poudre Dentaire

Marchande de Citrons Verts

Marchande de Sel

Marchande de Lungi

Orfèvre
Propriétaire de Magasin de Papier de Rebuts

Rouleuse de Beedi (Cigarettes)

Fabricante de Gâteaux Secs

Menuisière

Commerce d'Impression sur Tissus

Propriétaire de Papeterie

Fabricante de Brosses

Magasin "pour passer le temps" (denrées alimentaires)

Marchande de Fleurs

Fabricante de Sacs en Fil de Fer

Couturière

Chargeuse de Charrette

Marchande de Cacahuettes

Propriétaire de Confiserie

Repasseuse Itinérante

Confection de Petits Pains et Gâteaux Marchande de Poudre de Café

Propriétaire de Magasin de Bicyclettes Fabricante de Boites en Carton

Marchande de Riz
Propriétaire de Magasin de Viande

Brocanteuse

Ferrailleuse

Enfileuse de Perles

Fabricante de Boites en Bois

Marchande de Bracelets pour

Chevilles

Tisserande de Paillassons

Marchande de Poudre de Piments

Rouges

Brocheuse

Fabricante de Guirlande de Fils d'Or

Propriétaire de Stand de Confiserie

Marchande d'Oeufs

Fabricante d'Ustensiles de Bois

Fabricante de Jouets

Marchande de Sacs en Jute

Propriétaire de Magasin

de Chaussures

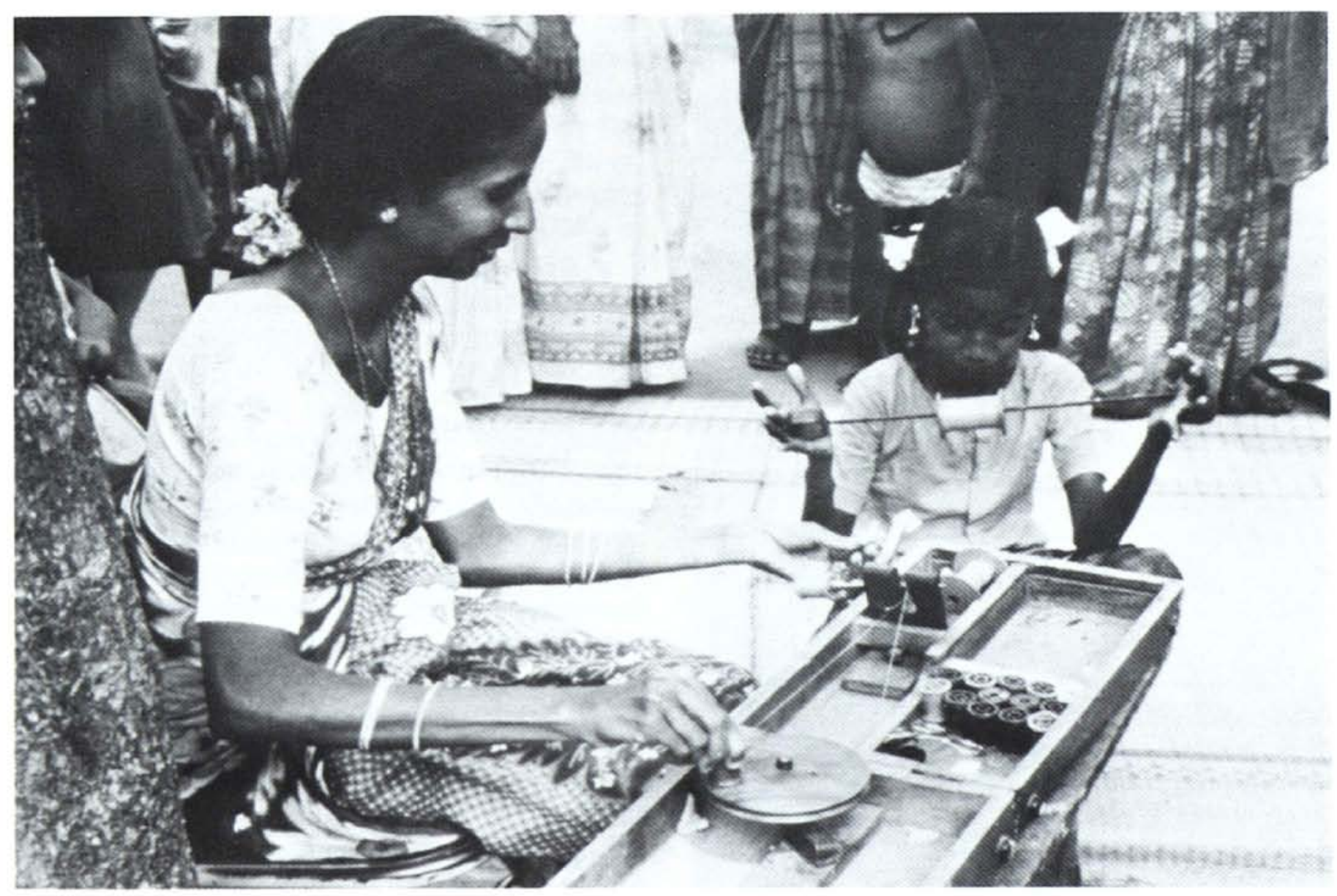


A l'intérieur de chaque catégorie les distinctions étaient encore plus subtiles. Certaines femmes travaillaient à la maison alors que d'autres se rendaient à un lieu de travail fixe; d'autres encore faisaient du colportage. Certaines femmes achetaient en gros et d'autres achetaient au détail. Certaines payaient comptant; d'autres achetaient à crédit. Certaines femmes se limitaient à vendre dans leur quartier, d'autres se rendaient sur des marchés locaux. Certaines femmes travaillaient à temps partiel tandis que d'autres parcouraient quotidiennement de longues distances pour vendre leurs produits. La plupart d'entre elles, pourtant, rencontraient d'énormes difficultés pour accomplir leur travail, des difficultés qui étaient pratiquement toujours liées à une faible productivité, un revenu modeste et un endettement perpétuel.

Les raisons pour lesquelles les femmes restent confinées aux niveaux les plus bas du commerce et des affaires sont variées et complexes. La structure de l'économie et du marché sont plus favorables aux hommes qui ont le monopole des marchés de gros, de l'achat de marchandises et du crédit. Les ferrimes sont fréquemment harcelées et écartées des marchés de gros car elles sont considérées comme un grand risque financier en raison du faible volume de leurs affaires. Or, pour développer une affaire, il faut avoir accès à des matières premières et des fonds plus importants. II n'est donc pas étonnant que les entreprises des femmes ont du mal à croitre. Beaucoup de femmes sont, en outre, limitées dans leur déplacement en raison des coutûmes sociales ce qui les empêche de se lancer dans des activités plus lucratives. D'autres sont limitées par le temps qu'elles peuvent consacrer à leur affaire en raison de leur lourde responsabilité domestique.

\section{L'adhésion au Forum}

La raison principale pour laquelle les femmes adhèrent au Forum est l'accès au crédit. En effet, les prêts qui leur sont consentis à travers le Forum sont plus élevés que ceux accordés préalablement par les prêteurs locaux et à un taux d'intérêt raisonnable. L'accès au crédit a considérablement changé la vie de plus de 7000 femmes qui ont, jus-

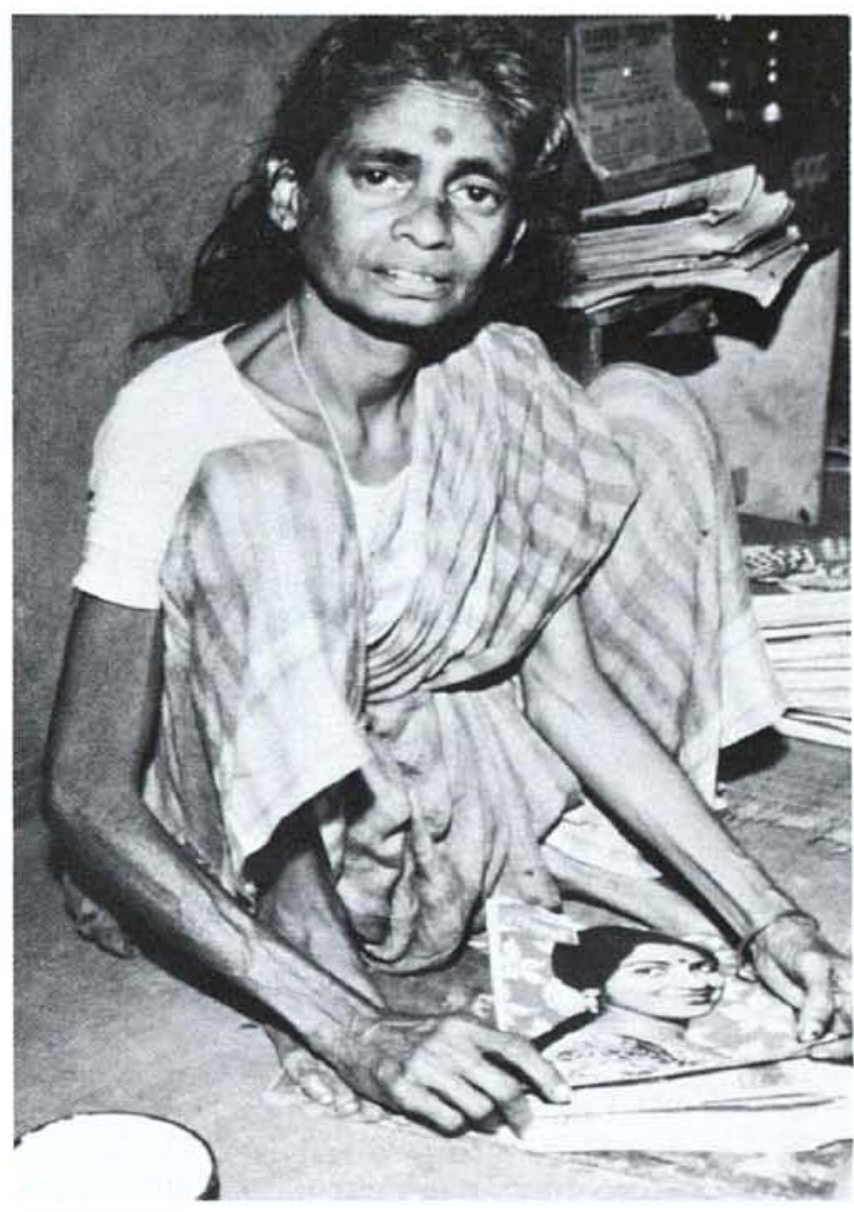

qu'ici, obtenu des prêts par le biais du Forum, comme nous le montre l'exemple, ci-dessous:

Kamala tient un petit stand de galettes devant sa maison. Le matin, elle vend des galettes de riz et de lentilles à la vapeur et, l'après-midi, des galettes de riz frites. Une fois par semaine, elle achète du riz et des lentilles et, chaque soir, elle les mouds pour en faire de la farine et préparer la pâte qu'elle doit laissée reposer pendant la nuit. Auparavant, Kamala devait acheter ces marchandises à crédit et payer un taux d'intérêt journalier. En remboursant ce qu'elle devait avec les intérêts, elle gagnait environ 50 cents par jour. A présent, grâce au prêt obtenu à travers le Forum pour les Femmes Actives, Kamala peut acheter les marchandises en gros, sans payer d'intérêt élevé et elle gagne USD 1 par jour.

Dans le quartier de Kamala, une centaine de femmes tiennent un petit commerce similaire au sien et vendent des denrées alimentaires. Des centaines d'autres vendent des 'légumes ou du kérosène, ou mercerisent du fil (c'est-à-dire imprègnent le fil d'une solution de soude caustique pour en faciliter la teinture) ou 
roulent des boulettes de charbon de bois et de fumier dont on se sert comme combustible de cuisson. Certaines femmes des bidonvilles travaillent pour de grands commerçants et sont rémunérées à la pièce, d'autres touchent un salaire fixe. Dans certains ménages, le mari et la femme travaillent tous deux dans la même entreprise. Dans la majorité des cas, pourtant, les femmes sont indépendantes et ont une source de revenu propre.

\section{Fonctionnement du Forum}

En dépit du nombre élevé de ses membres, l'organisation du Forum est relativement simple. Un des aspects peut-être les plus remarquables est que l'ensemble des cadres et du personnel administratif, à l'exception de Jaya Arunachalam, sa présidente-fondatrice, sont d'anciens membres du Forum, autrement dit, des femmes pauvres, pour la plupart illettrées venant des bidonvilles. Ces femmes ont toutes été sélectionnées en raison de leur esprit d'organisation que certaines avaient acquises en travaillant avec des partis politiques. De façon générale, les membres du personnel ne suivent pas de stages de formation en gestion ou en management mais apprennent sur le tas, par expérience.

Les responsables du Forum ont développé leur organisation en appliquant les principes de l'organisation politique. L'accent est plus particulièrement mis sur les gens à la base. Une organisatrice locale pour environ mille personnes travaille directement dans la communauté. Constituer de nouveaux groupes et former de nouveaux responsables sont une préoccupation constante pour s'assurer que les orientations et la politique à suivre du Forum restent l'affaire des membres à la base.

La structure de base de l'organisaton fut établi en fonction des exigences requises par le programme de crédit. (Voir l'Organigramme en Annexe I).

\section{Fonctionnement du Programme de Crédit}

Lorsque le gouvernement d'Inde nationalisa les banques, il prescrit un taux d'intérêt différentiel (TID) de 4\% par an pour les "segments les plus faibles de la société". Ce programme est plus connu sous le nom de plan pour le "petit emprunteur". Bien que, en théorie, les femmes actives de Madras qualifiaient pour le TID, elles n'obtenaient pratiquement jamais de crédit des banques. Les prêteurs locaux ou leurs proches étaient les seules sources de crédit disponibles avec un taux d'intérêt de 120\% par an! Lorsque Jaya Arunachalam défia les banquiers de faire quelque chose pour aider les femmes pauvres, ils durent admettre que pour eux le TID s'appliquait seulement aux hommes pauvres.

Les femmes elles-mêmes disaient que les banques hésitaient à leur accorder du crédit parce que, pour ces dernières, les petits commerces des femmes ne constituaient pas d'entreprises légitimes et elles n'aimaient pas financer une quantité innombrable de petits prêts. Les banques exigaient également qu'un homme contresigne la demande de prêt. En outre, les femmes hésitaient à se rendre auprès des banques parce qu'elles les trouvaient peu serviables et impersonnelles, rarement enclines à aider les emprunteuses illettrées à remplir les nombreux formulaires requis pour obtenir un prêt.

Afin de pallier ces problèmes, les fondatrices du Forum décidèrent d'organiser les emprunteuses potentielles en groupes de prêt à garantie mutuelle qui, par le biais d'un processus administratif efficace, les mettraient en contact avec les banques nationalisées.

Groupes de Prêt de Quartier. Le groupe de prêt de quartier est l'élément fondamental dans la structure du Forum. Toute personne désireuse d'adhérer au Forum doit appartenir à l'un de ces groupes. Chaque groupe est composé de 10 à 20 femmes venant toutes du même quartier. La plupart des groupes se forment par le bouche à oreille.

Les membres et le personnel du Forum tiennent des réunions de groupe et des discussions à intervalle régulier pour familiariser les nouvelles venues avec le programme. Généralement, une responsable de groupe potentielle contacte une employée du Forum. Cette dernière lui demande alors de constituer un groupe de 10 à 20 femmes et lui explique comment fonctionne le Forum. Lorsque un nombre suffisant de femmes sont réunies, elles élisent une responsable de groupe. Quelquefois aussi, une employée du Forum se met 


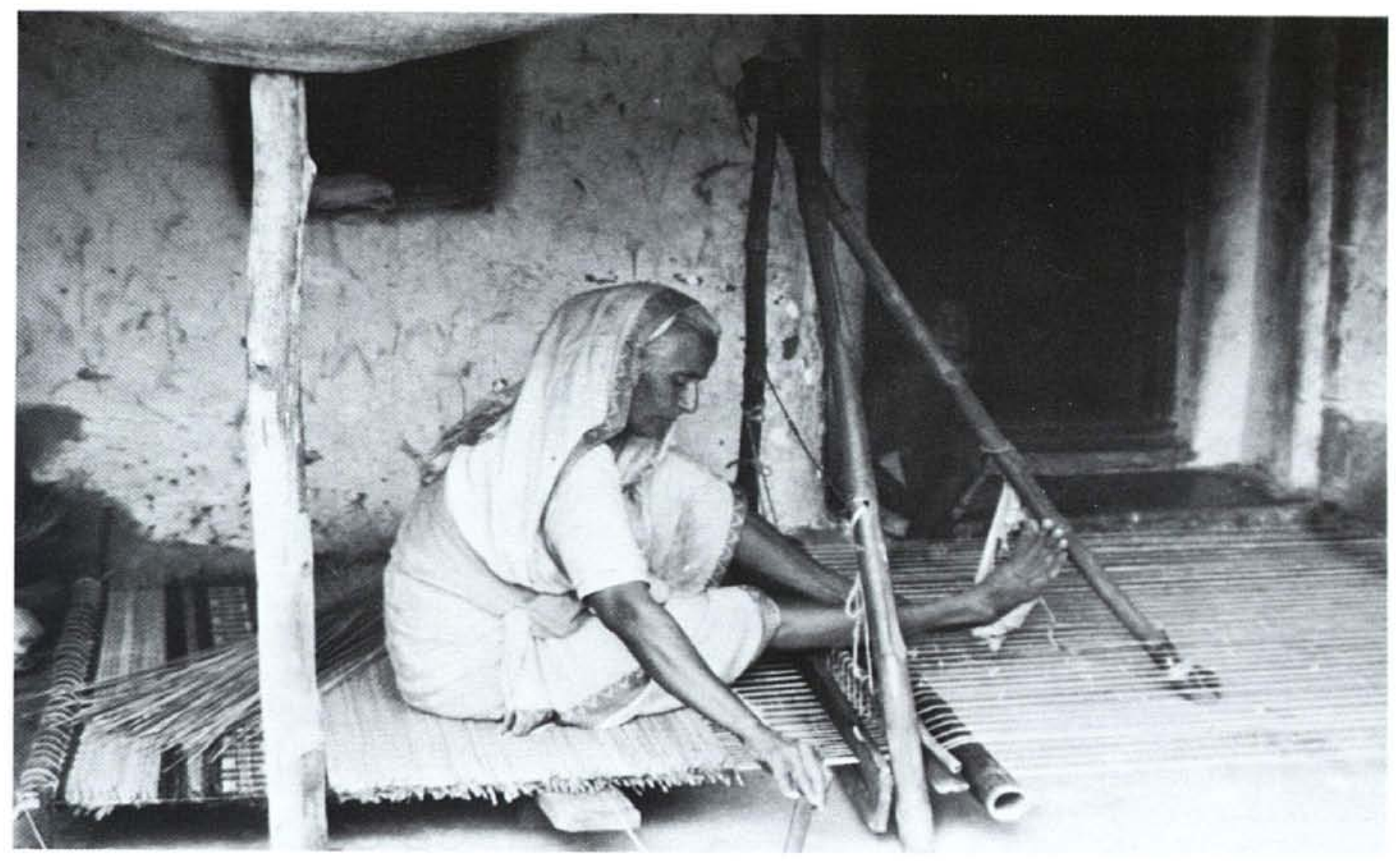

directment en contact avec une femme et l'encourage à constituer un groupe.

Une fois que le groupe de quartier est constitué, on l'inscrit au registre du Forum. Chaque membre remplit alors une demande d'inscription et verse une cotisation annuelle. Une femme peut devenir membre titulaire ou membre associé. Les membres titulaires versent une cotisation annuelle de 12 roupies (USD 1,33), ce qui leur permet de participer au programme de crédit. La plupart des femmes deviennent des membres titulaires. Les membres associés versent la moitié des frais de cotisation mais n'ont pas le droit de participer au programme de crédit.

Les obligations des membres sont simples: un membre doit participer régulièrement aux réunions de groupe, rembourser les prêts à intervalle régulier, et se porter garant pour les emprunts de tous les autres membres du groupe.

Procédure d'obtention d'un prêt. Une fois inscrits au Forum, les membres du groupe peuvent faire une demande de prêt. Tous les membres déposent leur demande en même temps. Le groupe a la possibilité de déposer une demande pour obtenir un sec- ond prêt lorsque le premier a été remboursé dans son intégralité. Les différentes étapes pour obtenir un prêt sont les suivantes:

\section{Evaluation de la Solvabilité}

- Avant de recommander les membres du groupe à l'organisatrice locale du FFA pour un prêt, la responsable procède à une évaluation minutieuse des besoins, des capacités de remboursement et de la productivité de chaque membre.

- Les membres du groupe évaluent la solvabilité de chaque membre du groupe avant de se porter garant mutuel ou caution.

\section{Demandes de Prêt}

- La responsable du groupe et les mem. bres retenus s'adressent à l'organisatrice locale du Forum.

- La responsable du groupe, les membres et l'organisatrice locale du FFA se rendent au bureau du Forum pour déposer la demande.

- Au bureau du Forum, chaque membre remplit une demande de prêt (un formulaire simple d'une page) avec l'aide du 
responsable du groupe, de l'organisatrice locale, du secrétaire général et de la responsable des prêts.

- Le secrétaire général et la responsable des prêts trient les demandes et les soumettent aux succursales bancaires concernées (à présent, le Forum travaille en collaboration avec huit succursales de la Banque d'Inde).

- Le secrétaire général et la responsable des prêts indiquent à l'organisatrice locale à quelles succursales les demandes individuelles ont été soumises.

\section{Versement des Prêts}

- L'organisatrice locale contacte les succursales respectives pour savoir à quelle date les prêts seront versés.

- L'organisatrice locale emmène les membres à la banque à la date stipulée.

- Là, assistés par un responsable de la banque, les membres remplissent chacun deux formulaires; le responsable de la banque remplit alors le reste des formulaires (cette étape prend au moins une demi-journée par groupe).

\section{Remboursement des Prêts}

- Les prêts sont accordés moyennant un TID de 4\%.

- Les prêts sont remboursables en dix versements mensuels (on a préféré dix versements à douze pour faciliter les calculs).

- La responsable du groupe est chargée de la collecte et du dépôt des paiements mensuels à la banque. Elle collecte l'argent auprès des membres une fois par jour ou une fois par semaine selon la préférence de chacune.

- L'organisatrice locale n'intervient qu'en cas de défaut de paiement de l'un des membres.

\section{Le Crédit du Point de Vue des Femmes}

Les banques nationalisées stipulent qu'il faut investir les prêts dans des activités "productives" (par exemple, des investissements dans le commerce ou les affaires) et la plus grande partie de l'argent que les femmes empruntent va directement dans leur affaire. Pourtant, la majorité des femmes utilisent également une portion de leur prêt pour subvenir à des besoins de consommations de première nécessité (nourriture, habillement, ustensiles de ménage ou réparation de leur maison) ou pour rembourser des dettes. En temps qu'intermédiaire entre les femmes actives et les banques, le Forum a eu la possibilité de mettre en place un système de remboursement flexible qui tient compte de ces réalités.

En effet pour le Forum, la notion "d'activités économiques et productives" revêt un caractère plus large et comprend également le travail à domicile et la garde des enfants. Le Forum admet également qu'un emprunt puisse servir à financer l'achat de matières premières ou couvrir des frais médicaux. II en va de même pour les dépenses à caractère social tels que les mariages ou les festivités religieuses qui revêtent une importance considérable dans la culture indienne et que les femmes considèrent comme des investissements économiques et sociaux. Le Forum reconnait également l'importance de s'acquitter de ses dettes auprès des prêteurs locaux ou des fournisseurs puisque cela permettra éventuellement à une femme de mettre plus d'argent dans son affaire. En outre, cela lui permettra de rester en bons termes avec le prêteur qui n'hésitera pas à lui prêter de l'argent en cas de besoin.

Décider qui recevra un prêt et qui pourra remettre un versement à une date ultérieure ou modifier le calendrier de remboursement revient à la responsable des prêts du Forum. Les raisons permettant à une femme d'ajourner un ou plusieurs versements-que la communauté bancaire qualifierait d'exceptionnellessont tout à fait justifiées et tiennent compte des réalités économiques des femmes pauvres. Ces raisons sont les suivantes:

- Fluctuations exceptionnelles et imprévues de la production et du cours des matières premières lorsque, par exemple, les rues et les maisons sont inondées à cause de la mousson ce qui affecte la production et le prix des denrées périssables, les moyens de transport et les communications. 


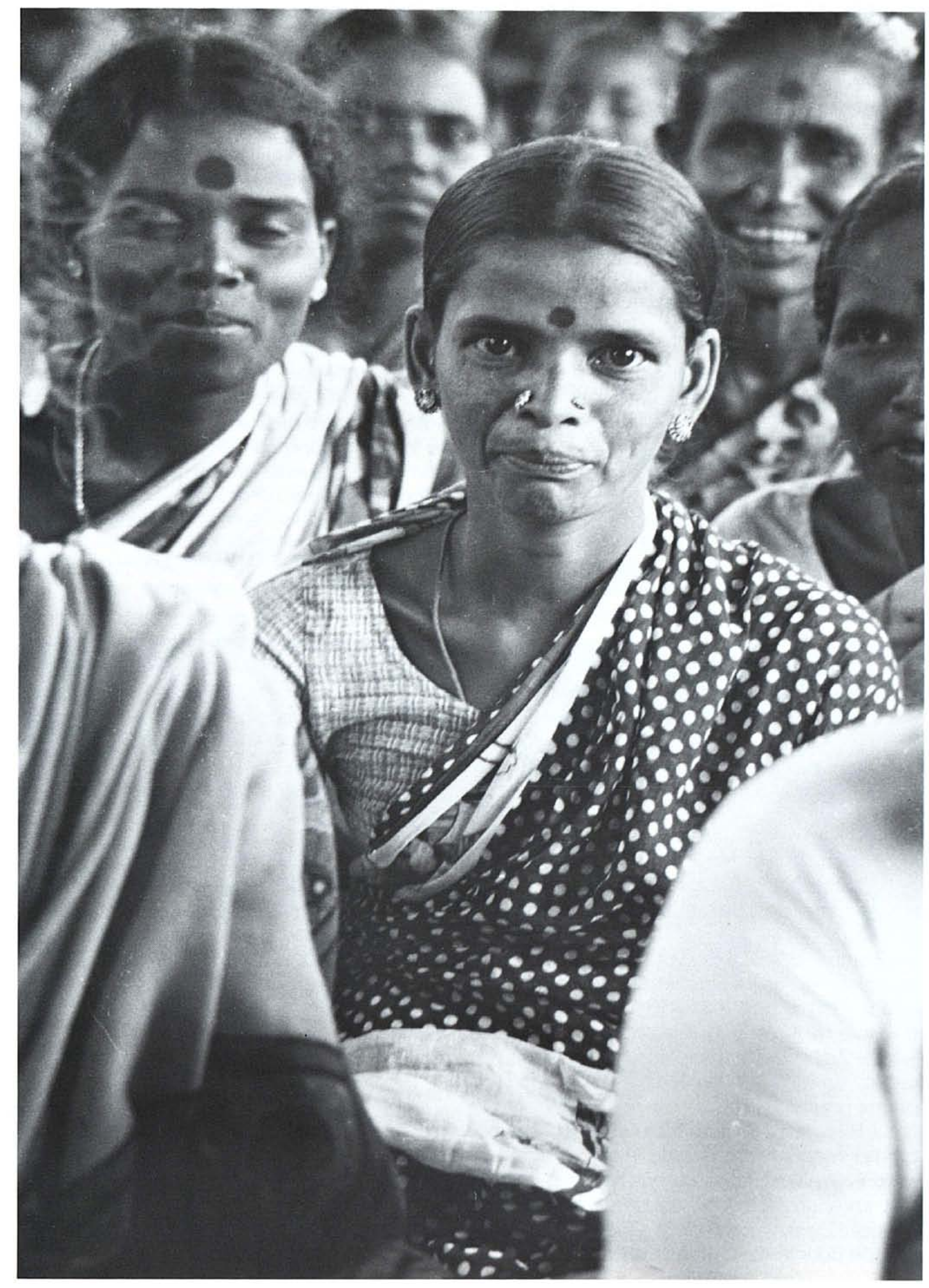


- Mariages. En fait, il s'agit d'une transaction économique puisque la famille de la mariée doit investir une somme d'argent convenable pour s'assurer d'un bon mariage et les deux familles doivent investir beaucoup de temps, d'énergie et d'argent pour préparer les diverses cérémonies de mariage.

- Naissances. Bien que les femmes reprennent leur travail quelques jours (ou même quelques heures) après l'accouchement, il y a tout de même des frais (par exemple, la sage-femme) et une perte de temps (allaitement, soins du nourrisson) à prendre en considération.

- Traitements médicaux. Lorsqu'une femme doit se rendre à un centre de santé pour un certain temps (par exemple, en cas de maladie grave, de stérilisation, etc...) ou amener un enfant ou un parent malade, cela lui fait perdre des journées de travail.

- Accidents et catastrophes. Les habitants des bidonvilles sont fréquemment victimes d'incendies et d'innondations, ce qui bien souvent les laissent sans provisions ni argent.

- Fêtes. Les fêtes religieuses tiennent une place importante dans la vie des femmes. Ils sont, non seulement, source d'inspiration et d'espoir pour les femmes mais aussi très souvent leur seule occasion de se divertir.

Lorqu'un membre a une "bonne raison", on lui permettra d'ajourner un ou deux versements. La plupart de ces "bonnes raisons" ont un caractère saisonnier et un programme flexible comme celui du Forum est en mesure de les prendre en considération. En cas de décès, les banques considèrent que le défaut de paiement est légitime. Pourtant, si une femme âgée veut participer au programme de crédit, un de ses héritiers devra se porter garant pour son prêt.

Bien sûr, il y aura toujours des femmes qui ne s'acquitteront pas de leurs dettes sans raisons valables. Ainsi, lorsque le Forum venait tout juste de démarrer, certaines mauvaisespayeuses essayèrent de s'affilier à un autre groupe pour tenter d'obtenir un second prêt. A présent, dans de pareilles situations, le Forum met fin à l'adhésion de la débitrice et lui reverse le montant de sa cotisation. Le Forum donne toutefois une seconde chance à toutes celles qui apportent la preuve de s'être véritablement réformées. De temps à autre, une responsable de groupe malhonnête tentera de garder pour elle-même les versements des autres membres. Pourtant, lorsque l'avis d'avoir à payer de la banque est porté à l'attention du groupe, les femmes pourront faire pression sur la responsable pour l'obliger à rembourser la somme qu'elle a détournée. En fait, tout le système repose sur la pression que les membres exercent les uns sur les autres. Le groupe fait pression sur chaque membre pour les obliger à s'acquitter de leurs dettes puisque si un individu ne paie pas, la réputation de solvabilité de tout le groupe est en jeu. Pareillement, les membres peuvent faire pression sur la responsable pour l'amener à exercer ses responsabilités honnêtement et sérieusement.

\section{Résultats du Programme de Crédit}

Le programme de prêts a eu un impact très tangible sur les affaires de ces femmes et sur le bien-être de leur famille. Grâce à lui, environ 2800 nouveaux emplois ou petites entreprises ont été créés et les petits commerces existants ont augmenté leur profit en moyenne de $50 \%$. Ce programme a également permis aux femmes de développer et de diversifier leurs activités économiques.

Au début, Ramanji vendait des légumes. Elle utilisa le premier prêt (Rs 100 soit USD 11) pour investir dans son affaire. Elle utilisa le deuxième prêt (Rs 200) pour rembourser toutes les dettes qu'elle avait contractée dans l'exercice de son commerce. Avec le troisième prêt (Rs 300) et Rs 500 qu'elle emprunta d'une autre source, elle décida d'investir dans une autre affaire : la production de brosses en toile pour polir le métal. Elle emploie, à présent, quatre autres femmes et a abandonné la vente de légumes pour se consacrer à plein-temps à la confection de brosses. Elle a l'intention de demander un autre prêt de Rs. 1000 pour acheter une machine à coudre.

Le mari de Ramanji a un travail temporaire comme peintre. Ses trois fils sont au chômage. Ramanji est la seule à rapporter chaque jour un salaire régulier. 
Grâce au programme de crédit et au soutien moral du Forum, un nombre important de femmes ont investit dans une affaire pour la première fois.

Avant d'adhérer au Forum, je travaillais comme coolie sur des chantiers. C'est là que j'ai rencontré Pattammal (une des responsables du FFA). Elle m'a demandé comment çà allait et je me suis décidée à lui confier tous mes problèmes. Elle m'a alors conseillé d'adhérer au Forum et m'a dit que je pourrais obtenir un prêt pour démarrer une petite affaire. Je me suis inscrite, j'ai obtenu le prêt et j'ai, à présent, un petit commerce de vente de tissus.

Murugammal, Membre

Les femmes elles-mêmes signalent que l'indicateur le plus fondamental du succès du programme est qu'elles mangent mieux; des familles qui, très souvent, devait se contenter d'un repas par jour peuvent, à présent, en avoir deux et mangent de la nourriture plus variée et de meilleure qualité. Une étude sur la manière dont 300 bénéficiaires de prêts investissaient leurs gains supplémentaires révéla les priorités de dépenses suivantes: nourriture, habillement, épargne, éviter les prêteurs locaux, ustensiles de ménages, médicaments, meilleure éducation pour les garçons, réparations de la maison, meilleure éducation pour les filles, et bijoux (les femmes indiennes n'investissent pas en bijoux par vanité mais parce qu'ils constituent une sécurité financière pour le futur).

Depuis leur adhésion au Forum, la plupart des femmes ressentent une plus grande sécurité financière. Pour la première fois, leurs activités économiques sont consignées dans les annales d'une institution financière réputée et elles sont enfin reconnues pour leur travail. C'est aussi la première fois que les femmes pauvres ont pu obtenir un prêt sans qu'un homme se porte garant ou contresigne la demande.

Nous avons totalement réussi à éliminer le fait que les hommes de la famille se portent garant pour les femmes. Elles jouent désormais un rôle économique à part-entière.

Jaya Arunachalam

- Hilde Jeffers. Organizing Women Petty Traders and producers. A Case Study of the Working Women's Forum. Madras: WWF.

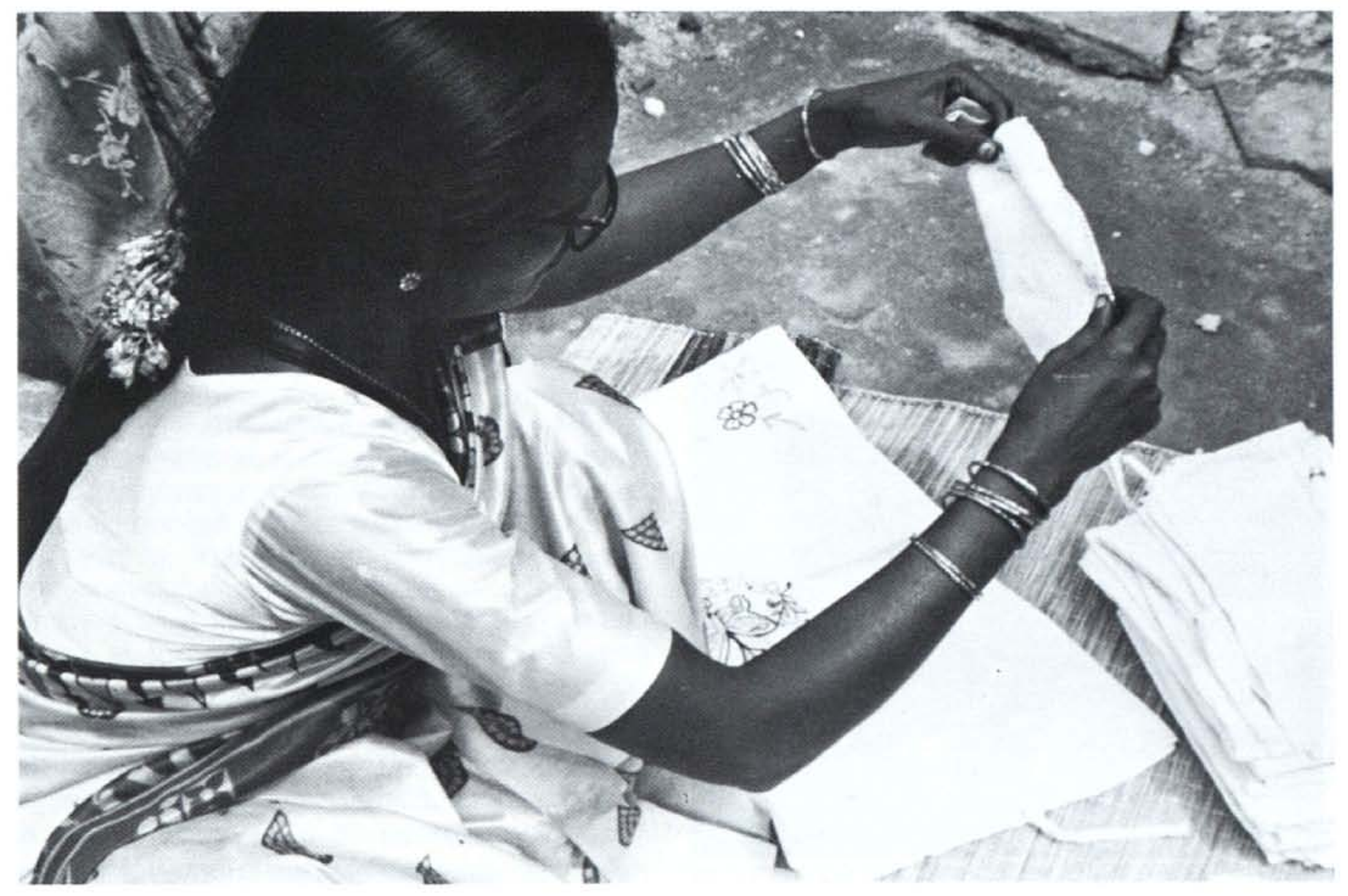



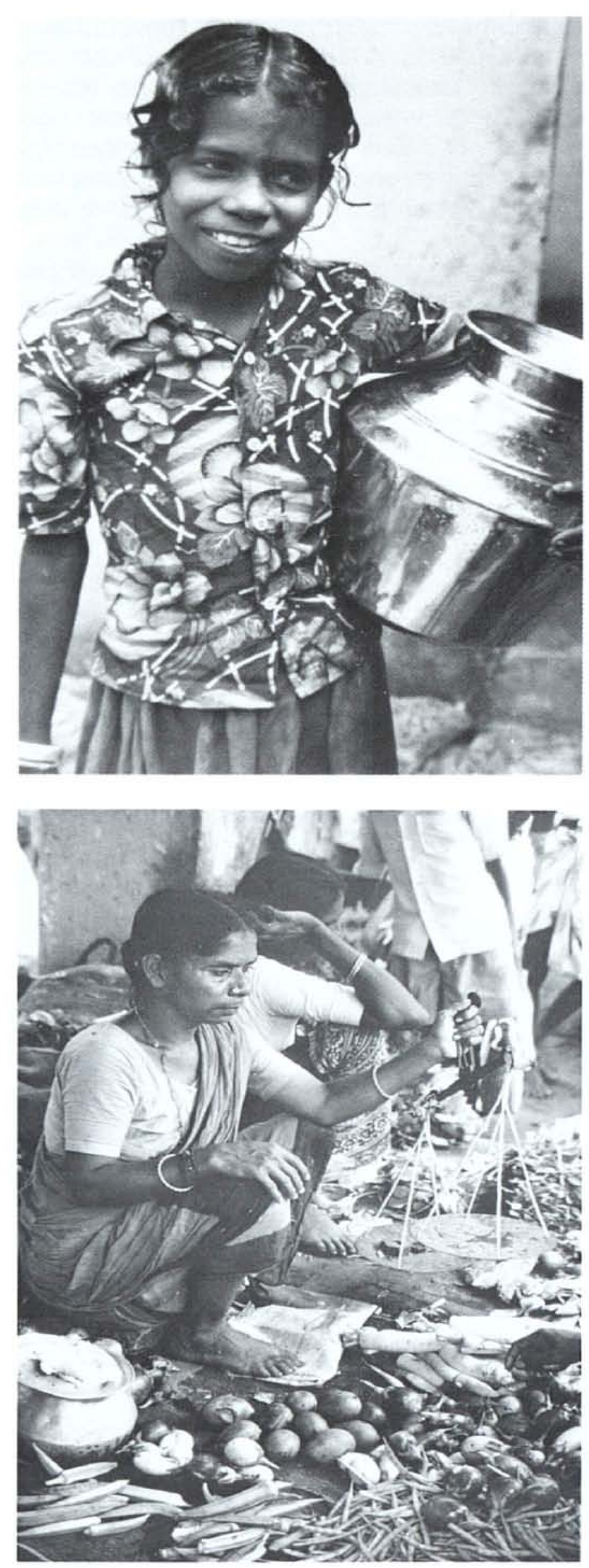

\section{Soutenir les Rôles Economiques Joués par les Femmes}

Pour le personnel du Forum, le programme de crédit est la première étape d'une stratégie plus large visant à apporter un changement social, une stratégie qui, progressivement, irait de la satisfaction des besoins économiques de base vers la résolution de problèmes politiques et sociaux beaucoup plus complexes auxquels les femmes sont confrontées. A présent que les groupes de prêt de quartier sont en place, il est possible d'entamer d'autres activités et programmes.

\section{Services de soutien.}

Un certain nombre de services sociaux ont été mis en place, la plupart d'entre eux à l'initiative des membres. Dans certains quartiers des bidonvilles, plusieurs groupes de prêts se sont associés pour mettre en place des programmes comme ceux décrits ci-dessous:

Garderie d'enfants. Le Forum considère que, pour les femmes actives et leur famille, la mise en place de crèches est une nécessité. Les groupes du Forum ont déjà ouvert dix centres. Des membres sachant lire et écrire servent d'enseignants et sont rémunérés par le groupe qui, à son tour, supervise leur travail. Ci-dessous, un enseignant relate le déroulement d'une journée typique à la crèche:

Trente-six enfants entre deux et cinq ans viennent à la crèche. Ils restent au centre de 9 heures à 15 heures. Une bonne (rémunérée par le Forum) et une mère (à tour de role) m'aident à nettoyer, huiler et peigner les cheveux des enfants. Après les prières, je fais l'appel et je leur enseigne le Tamil (la langue locale), l'anglais et les maths. Pour le déjeuner, nous leur préparons un repas nourrissant et bon marché constitué de lentilles, de légumes et de riz ou de salade, de tomates et de riz, avec du pain; entre les repas, nous leur donnons des casse-croûtes. Les mères nous aident à préparer le déjeuner. Les enfants font la sieste après le repas.

Très souvent ces centres sont situés dans de modestes locaux en chaume. Trouver de l'espace pour ouvrir davantage de centres s'est avéré difficile et c'est aussi la raison essentielle pour laquelle cette activité n'a pas 


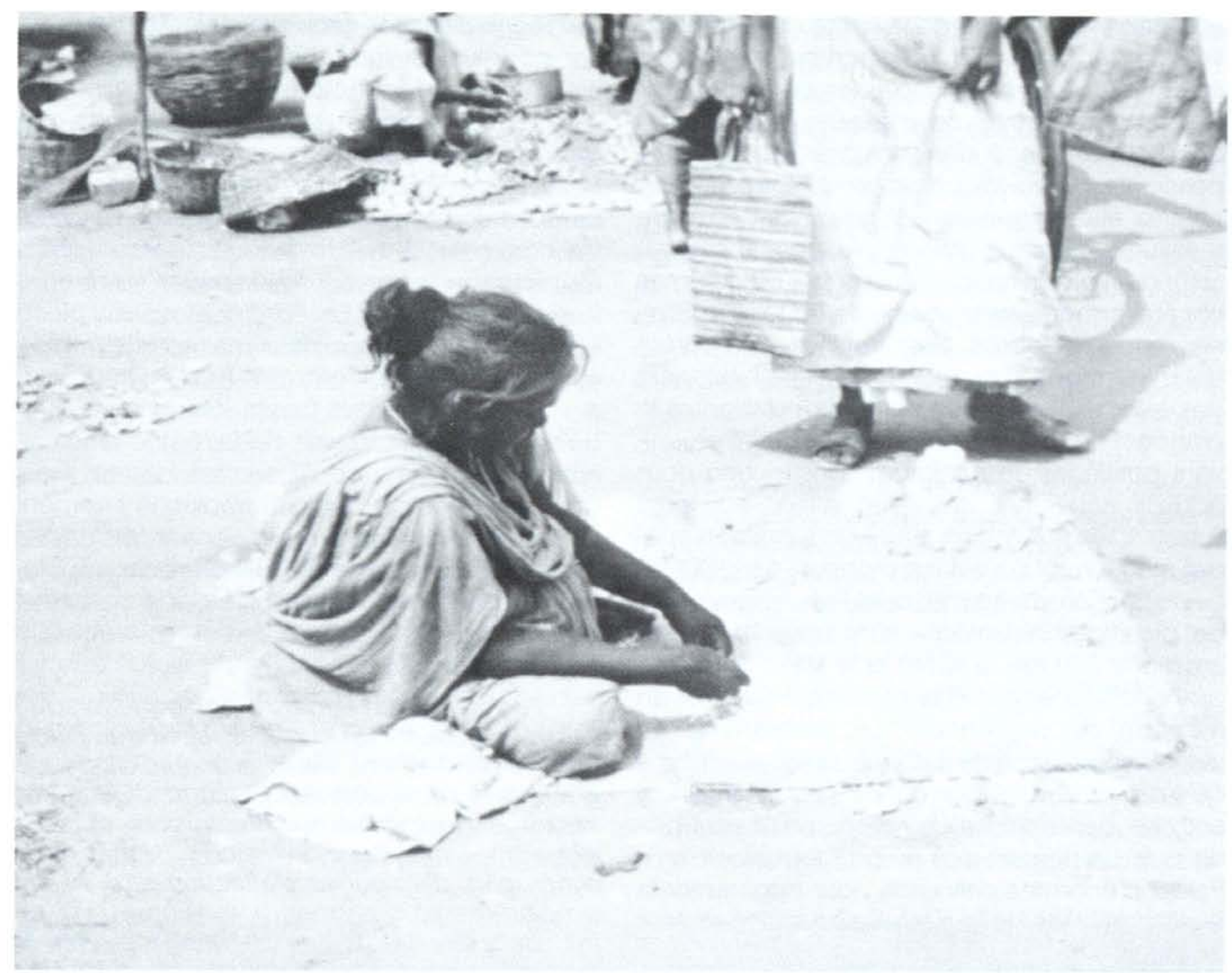

connu de développement plus poussé. Résoudre ce problème d'espace, et donner aux enfants une instruction et des soins de meilleure qualité, tout en mettant à leur disposition plus de jouets, constituent un objectif essentiel pour le Forum.

Cours du Soir. Les enfants allant à l'école primaire et au collège ont la possibilité de suivre des cours de rattrapage le soir. 22 classes, constituées, en moyenne, de 35 à 50 garçons et filles, viennent d'être ouvertes. Comme pour les crèches, les enseignants sont des membres du groupe qui savent lire et écrire. Ils sont rémunérés par le groupe qui, à son tour, supervise leur travail. Le but de ces classes est d'améliorer les résultats scolaires des enfants et d'éviter ainsi qu'ils n'abandonnent l'école. Les enseignants aident les enfants avec leur devoir et leur donnent des leçons particulières dans les matières dans lesquelles ils sont faibles. Les résultats scolaires des enfants se sont considérablement améliorés à la suite de ces cours du soir.

Centres de Formation. Le Forum a ouvert dix centres de formation pour permettre à ses membres de se recycler ou de perfectionner leurs aptitudes professionnelles. Ces centres sont destinés aux chômeuses et à toutes celles qui occupent des emplois très mal rémunérés. A la fin du stage, toutes celles qui ont l'intention de postuler à des emplois dans des industries locales (notamment dans la confection) reçoivent un diplome de fin de stage. D'ici quelque temps, le Forum a l'intention de mettre en place un programme pour aider les futures diplomées à se constituer en coopératives de production.

Santé et Planification Familiale.

En 1980, le Forum a mis en place un programme expérimental de formation en ges- 
tion dans le domaine de la santé et de la planification familiale. Ce programme visait, non seulement, à faire bénéficier les communautés de soins de santé de meilleure qualité mais encore à donner à des membres du Forum la possibilité de suivre un stage de formation en gestion et en management pour leur permettre d'assumer de plus grandes responsabilités au sein de l'organisation. Jusqu'ici, 60 femmes ont été formées comme agents de terrain. Elles se rendent auprès des familles demeurant dans leurs quartiers respectifs et leur donnent des informations sur la santé, la nutrition et la planification familiale. Toutes les stagiaires doivent posséder un minimum d'instruction pour pouvoir consigner des informations sur leurs visites. Elles suivent un stage de formation spécial et touchent un salaire mensuel de USD 18.

Le Forum estime que les programmes de planification familiale sont essentiels pour améliorer à la fois la santé et le statut économique des habitants des bidonvilles. Pour citer un membre du personnel: "Le programme de planification familiale fut mis en place parce qu'il devint évident que familles nombreuses et activités génératrices de revenus n'allaient pas de pair. La plupart des emprunteuses rencontraient d'énormes difficultés pour augmenter la qualité de la vie de leur famille en raison de leur grande taille".

Le programme de santé et de planification familiale a eu un impact considérable sur la communauté comme nous le laisse entendre une stagiaire en parlant de son quartier:

Les habitants des bidonvilles n'ont jamais su comment parler aux "autres". Mais après m'être inscrite au FFA, j'ai appris beaucoup de choses. Auparavant, on ne montrait jamais les enfants (on les gardait cachés) lorsque les gens de la corporation (santé publique) venaient pour les vacciner. Après notre stage de formation, on nous a dit de visiter 100 maisons chacune. Après leur avoir expliqué les bienfaits de la vaccination, ils ont emmené les enfants pour se faire vacciner.

\section{Marayammal, Agent de santé et de planification familiale}

\section{Action Sociale.}

L'organisation et la mobilisation sont l'essence même du Forum pour les Femmes Actives. Le Forum s'est fondé sur la cohésion qui règne dans les groupes de prêts de quartier pour unifier les femmes autour de questions qui, traditionnellement, auraient pu les diviser (caste, religion, politique). En temps que groupe, elles se sentent plus à l'aise pour confronter les forces politiques qui seraient perçues comme une menace si elles devaient les confronter à titre individuel (responsables municipaux, police, revendeurs, prêteurs locaux, grossistes). Le Forum a mis en place les activités suivantes pour maintenir et renforcer la cohésion des groupes locaux:

Mariages Inter-Caste, Sans Dot. Bien qu'en Inde, il soit interdit de faire une discrimination entre gens de différentes castes, il est difficile de venir à bout de traditions tellement anciennes. Pour les femmes venant des castes inférieures cela constitue un obstacle supplémentaire à surmonter en plus de la pauvreté, comme nous le décrit un membre en relatant sa propre situation:

Je ne parlais jamais à personne lorsque j'allais à l'école et les autres disaient que j'étais une Harijan *, d'une caste inférieure...Lorsque je disais à mon père en pleurant: "Certaines personnes me traitent différemment et sans respect", il me répondait alors: "Nous, nous avons plus d'amour et d'affection, eux ils ont simplement plus d'argent". Au Forum, on ne fait pas tellement de différence entre les castes.

Une autre coutume qui persiste toujours, bien qu'elle soit illégale depuis des décennies, est la dot. Le FFA a réussi à jeter le discrédit sur ces deux coutumes aberrantes en lançant une campagne en faveur des mariages intercastes. Plus de 300 membres du FFA se sont mariés sans dot et à peu de frais au cours de cérémonies de mariage de masses. Beaucoup de ces mariages servent simplement à légitimiser des unions de longue date et conférer ainsi aux femmes un statut social et légal. Des représentants du gouvernement assistent à ces cérémonies de mariage et le Forum saisit cette occasion pour promouvoir ses positions anticaste et anti-dot et pour pousser le gouvernement à tenir ses promesses et prendre enfin les mesures économiques nécessaires visant à faciliter les mariages inter-castes.

\footnotetext{
- Harijan signifie "enfants de Dieu". C'est le nom que Gandhi donna à tous ceux qui appartenaient aux classes sociales les plus défavorisées, et que I"on appelait les "intouchables", dans le but d'améliorer leur statut.
} 
Groupes de Pression en Faveur Des Biens et Services d'Utilité Publique. Le Forum aide ses membres à faire pression (et occasionnellement à se battre) auprès des responsables municipaux pour: réserver des emplacements aux femmes pour qu'elles puissent vendre leurs produits; construire sur les marchés des préaux en chaume pour permettre aux femmes de vendre leurs marchandises; construire des toilettes pour les femmes sur les marchés et autres endroits publics; construire et réparer les routes, toilettes, et maisons dans les bidonvilles; protéger les habitants des bidonvilles contre le harcèlement de la police.

\section{Impact}

La mobilisation des femmes autour de questions sociales et politiques ainsi que sur le crédit a profondément modifié leur attitude sur des sujets allant des castes à la planification familiale. A l'heure actuelle, la majorité des membres s'opposent à la pratique de la dot et approuvent les mariages inter-castes et sont, en outre, fortement convaincus qu'il est dans l'intérêt des femmes de jouer un rôle économique actif. La plupart d'entre elles pensent qu'il est préférable pour une femme d'exercer une activité professionnelle en dehors du foyer. Elles commencent également à réaliser qu'il leur est possible d'avoir un certain contrôle sur leur vie, y compris sur leur fécondité.

La plupart des membres du Forum signalent qu'elles ont gagné plus de respect et de pouvoir de décision non seulement dans leur propre foyer mais également au sein de la communauté. Elles se rendent compte que plus leur solidarité est grande, plus leur capacité de négocier et de revendiquer leurs droits augmente.

La majorité des femmes sont illettrées; certaines sont instruites mais la plupart d'entre elles sont très pauvres. Grâce à notre organisation, elles ont gagné la reconnaissance et le respect de leurs concitoyens... C'est un grand pas en avant pour les femmes.

Kala, Responsable de la Planification

Familiale

Il apparait, par conséquent, que les groupes de prêts de quartier sont bien plus qu'un simple système de prêts à garantie mutuelle. Ils sont également un lieu d'échange social pour

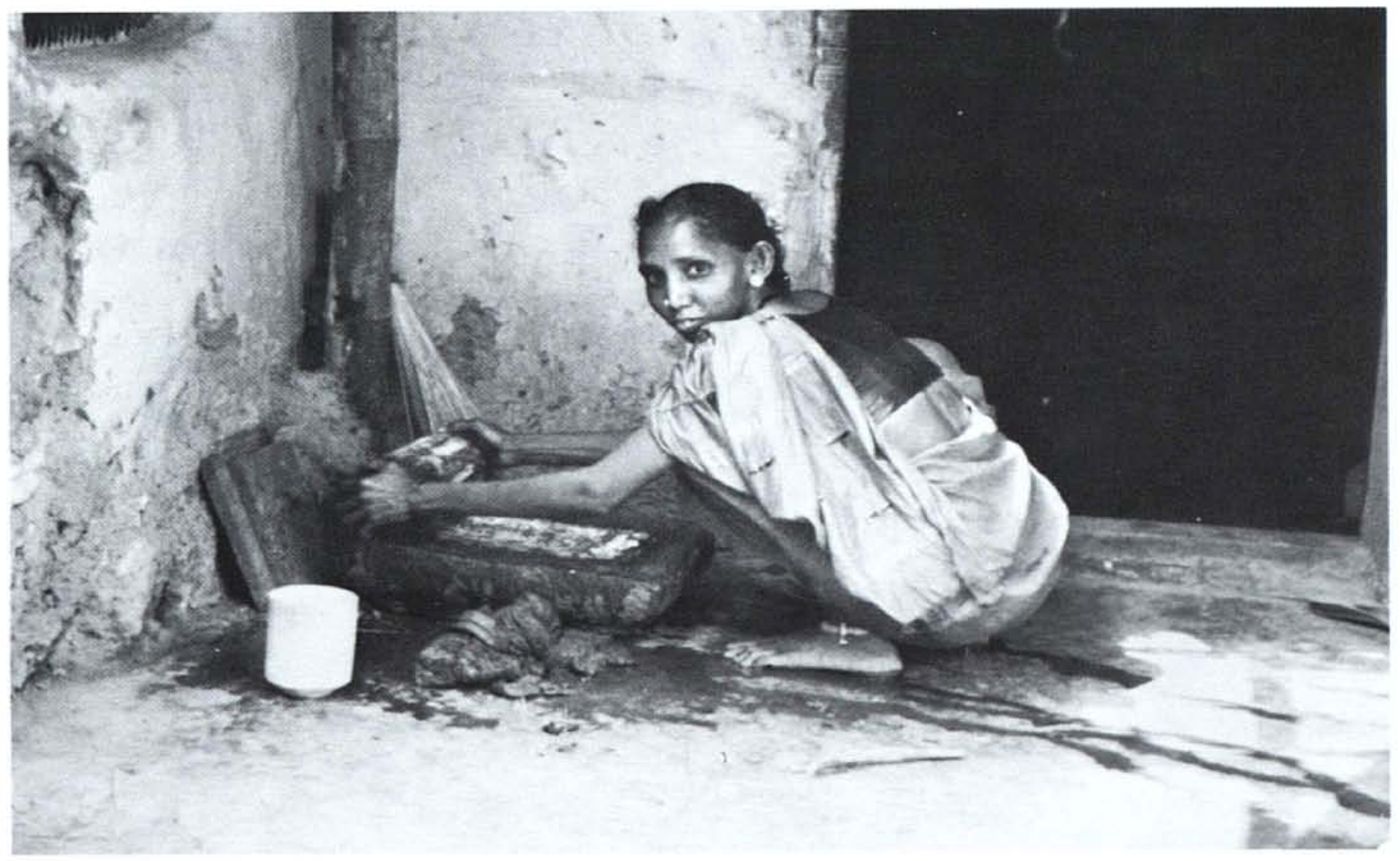


leurs membres. Très souvent, le groupe sera le premier endroit où les femmes créeront des liens en dehors de leur famille. C'est un lieu où elles ont la possibilité de discuter de leurs problèmes et de trouver des solutions. Par le biais de ces groupes, le Forum a réussi à faire monter à la surface le "pouvoir des femmes" jusque là en veilleuse.

\section{Nouveaux Développements}

Les banques nationalisées ont, jusqu'ici, accordé plus de 7000 prêts à des femmes pauvres par l'intermédiaire du FFA. Avec un taux de remboursement supérieur à $90 \%$, les banques sont très favorables aux groupes de prêt à garantie mutuelle et envisagent de poursuivre le développement du programme. Pourtant les choses ne sont pas si simples et, à I'heure actuelle, le Forum continue de rencontrer des difficultés graves dans le cadre de sa collaboration avec les banques nationalisées:

- Retard dans l'octroi des prêts en raison du nombre élevé de petits prêts ce qui veut dire que certaines femmes doivent continuer à emprunter de l'argent aux prêteurs locaux en attendant de recevoir leur prêt de la banque.

- Inflexibilité du calendrier de remboursement des prêts; le Forum a pu introduire les procédures de remboursement plus flexibles mentionnées précédemment en prenant en charge les frais soi-même.

En raison de ces difficultés, le Forum a décidé d'ouvrir sa propre banque en se fondant sur l'expérience acquise en gérant les programme de crédit initiaux. En 1981, le Forum a ouvert la "Société de Crédit Mutuel et de Services Sociaux pour les Femmes". Des membres du personnel soigneusement sélectionnés ont suivi un stage de formation spécial en administration bancaire et les emprunteuses ayant les meilleures réputations de solvabilité (5 000 sur un total de 13205 membres) ont été conviées à devenir actionnaire de la banque moyennant un montant de 20 roupies (USD 2,30) par action. Chaque actionnaire a eu droit à un crédit total équivalent à dix fois la valeur de l'ensemble des actions détenues dans la Société. Le Forum espère qu'en

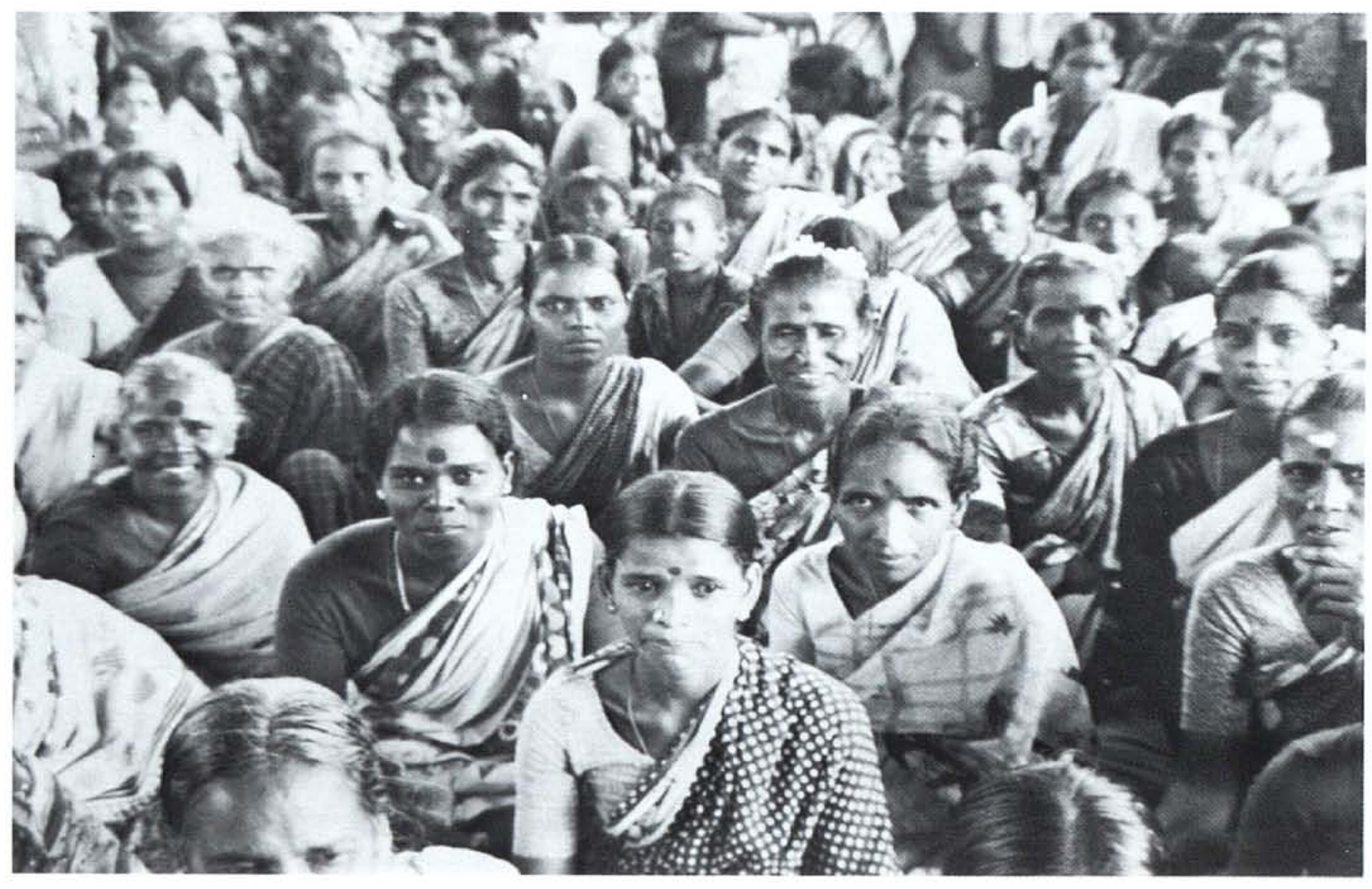


plus des conseils sur le crédit, la Société pourra, en son temps, donner des conseils en gestion et fournir de l'assistance technique à ses actionnaires.

\section{Syndicat National des Femmes}

Actives. En 1980, le Forum commença à travailler, non seulement avec des femmes vivant en milieu urbain mais également avec des femmes demeurant en milieu rural. Grâce à un programme gouvernemental appelé "Lab-toLand", le personnel du Forum lança un projet destiné à doter les femmes vivant en milieu rural de savoir-faire technologique. Le FFA aida 400 femmes ne possédant pas de terres à acquérir du bétail et à suivre un stage de formation en élevage. Le Forum aida également des pêcheuses et des dentellières vivant en milieu rural à s'organiser et à revendiquer des lignes de crédit auprès des banques.

Par le biais de ces activités, le personnel du Forum réalisa qu'en regroupant les femmes par catégorie professionnelle (non pas uniquement en fonction des liens de quartiers), il était possible d'augmenter leur pouvoir de revendication. C'est ainsi, qu'en Mai 1982, le Syndicat National des Femmes Actives fut crée, d'une part, pour poursuivre le développement des activités syndicales du Forum en toute légalité, et, d'autre part, pour sensibiliser les femmes travaillant à leur propre compte et "inorganisées" (par opposition à celles travaillant dans les usines où les ateliers) sur la nécessité de s'organiser en syndicat.

\section{Directions futures}

Lorsqu'on demanda à Jaya Arunachalam comment elle envisageait la direction future du FFA, elle foisonnait d'idées et de projets. En termes de stratégies générales, Jaya estime qu'il est nécessaire de :

- mettre en place des services sociaux supplémentaires dont les femmes ont besoin pour pouvoir accomplir pleinement leurs rôles économiques.

- fournir des services techniques plus complets et de meilleure qualité pour augmenter l'efficacité et la productivité des commerces tenus par les femmes;

- mettre en place des systèmes de marketing permettant de transformer les petits commerces des femmes en des activités commerciales plus florissantes;

- augmenter le pouvoir de revendication et renforcer les groupes de pression pour avoir plus d'accès et de contrôle sur les biens et services d'utilité publique.

En termes d'activités spécifiques, Jaya et ses collègues ont déjà formulé les propositions suivantes:

- création de coopératives de production pour garantir la sécurité de l'emploi et des emplois plus lucratifs, notamment, aux chômeuses et aux femmes occupant des postes très mal rémunérés;

- mettre en place des entrepôts où les femmes pourront acheter en gros des articles comme des saris, du tissus, des matières premières au prix le plus bas possible; et

- créer un réseau de marketing en gros pour relier les paysannes isolées, ne possédant pas de terres et habitant dans un rayon de $30 \mathrm{~km}$ de Madras, avec les commerçantes de Madras, et éliminer ainsi les revendeurs.

\section{Mise à Jour}

En 1983, le Forum pour les Femmes Actives avait la moitié de son âge et à peine un quart de sa taille actuels. Le Forum a non seulement mûri et connu un développement poussé, il a également changé sa conception de façon significative. Le FFA comprend à présent 60,000 membres et le montant total des prêts consentis à ses membres s'élèvent à plus de 13 millions de roupies (soit environ USD 1 million).

Le changement le plus significatif, à l'exception de l'augmentation considérable du nombre de ses membres et du volume des prêts, est qu'on ne peut plus caractérisé le Forum pour les Femmes Actives comme un projet urbain. En effet, vers 1983, le Forum avait achevé la mise en place de trois projets pilotes en milieu rural à l'intention des femmes s'adonnant au petit élevage, à la pêche et à la fabrication de dentelles. Ces projets ont connu un succès tel qu'en 1983 ils ont fini par devenir des branches à part-entière du Forum avec un nombre considérable d'adhérentes. Deux branches comprennent, à l'heure actuelle, plus 
de quatre mille membres et la troisième en compte plus de treize mille. Le Forum a également ouvert une quatrième branche en milieu rural et une seconde branche en milieu urbain en 1983; la première travaille avec des femmes qui roulent des cigarettes à la main et la seconde avec des fabricantes d'encens. Plus de la moitié des membres du Forum proviennent désormais de milieu rural.

Le Forum a également poursuivi ses efforts de syndicalisation des membres par secteur d'activités. L'établissement du Syndicat National des Femmes Actives a non seulement doté le Forum d'une identité légale, mais a également marqué un changement stratégique dans la manière dont le Forum était organisé. Pour la mise en place du programme de crédit initial et l'organisation préliminaire, le Forum avait misé sur les liens de quartiers pour édifier sa structure; avec la création du syndicat, il a commencé à organiser les femmes par catégorie professionnelle et à analyser les obstacles spécifiques aux femmes exerçant la même profession.

Depuis 1983, le Forum a également travaillé au renforcement de deux autres programmes. Le programme expérimental de planification familiale et de santé, lancé en 1980, a connu un développement et un impact considérables. Jaya Arunachalam, la fondatrice du FFA, voit les activités du Forum comme "un programme équilibré offrant des services de planification familiale/santé et de crédit/ emploi utilisant les mêmes structures locales et les mécanismes de quartier pour les dispenser". La Société de Crédit Mutuel et de Services Sociaux pour les Femmes, fondée en 1981, s'est également consolidée. Au cours de ces dernières années, les membres ont obtenu plus de prêts à travers la Société de Crédit du Forum qu'à travers les banques nationalisées. Sur un total de plus de 13 millions de roupies accordés comme prêts jusqu'en Mars 1987, plus de la moitié étaient financés par la Société de Crédit.

\section{Leçons à Retenir}

1. Les banques commerciales peuvent accorder des prêts très bas (aussi bas que USD 10) à un nombre important de femmes en étant garanti d'un taux de remboursement supérieur à 90\%. Cela peut se faire sans engendrer de frais généraux élevés, sans grande supervision, ni assistance technique.

2. Un programme de prêt peut être mis sur pied et développé rapidement si on rassemble en petits groupes (de 10 à 25) des femmes venant du même quartier ou ayant des occupations ou des intérêts similaires. Lorsqu'un programme de prêt est établi avec des institutions financières officielles, il est nécessaire de revoir le processus d'obtention des prêts de manière à confier l'évaluation de la solvabilité à des pairs et que les petites entreprises des femmes puissent servir de nantissement. De même, le système de remboursement des prêts devrait être fondé sur la pression que les membres exercent les uns sur les autres.

3. II est préférable de commencer un programme soutenant des activités économiques existantes plutot que d'essayer de recycler les femmes et de créer de nouveaux emplois. L'assistance technique, la formation et l'introduction de nouvelles activités peuvent être intégrées ultérieurement.

4. Un projet destiné à venir en aide aux femmes pauvres devrait démarrer avec des activités donnant des résultats rapides et tangibles. Mieux vaut résoudre leurs problèmes les plus immédiats et concrets. II est possible, par la suite, d'entamer des activités supplémentaires, allant de la résolution de leurs besoins économiques de base vers la résolution de problèmes sociaux et politiques plus complexes. Seules les questions dont les femmes discutent et qui sont au centre de leurs préoccupations devraient être retenues.

5. L'organisation d'un programme ne doit pas se faire suivant un plan élaboré dans ses moindres détails. Les besoins en ressources humaines et financières devraient émerger d'un programme en évolution constante.

6. Un programme pour les femmes est plus à même de réussir si on incorpore les deux éléments suivants: (a) une idéologie forte, profemmes, pour leur insuffler un esprit de solidarité et de la confiance en elles-mêmes, et (b) confier des responsabilités aux membres à la base pour renforcer et amplifier le pouvoir des femmes pauvres. 
7. II est préférable de s'appuyer, à chaque fois que cela est possible, sur des programmes gouvernementaux existants. Peu importe qu'ils existent réellement ou seulement sur le papier, ces programmes peuvent toujours être activés pour en faire bénéficier les femmes pauvres. Le Forum a démontré que cela est possible lorsqu'il a mis en place son programme pour "petits emprunteurs". En principe, il ne faudrait pas dupliquer de programmes existants. Pourtant, quelquefois il est nécessaire de créer des systèmes de prestations de services parallèles pour s'assurer que les femmes pauvres profitent des programmes établis.
8. Une organisation voulant atteindre et bénéficier un nombre important de femmes pauvres n'a pas besoin de fonds importants, de personnel éduqué ou d'expertise technique. Le succès du Forum est dû principalement aux quatres facteurs suivants: (a) sélection d'un thème crucial (crédit), (b) utilisation de responsables locaux, (c) regroupements des femmes venant du même quartier, et (d) gestion décentralisée et participation des membres aux prises de décision. Grâce a cette structure, le FFA n'a pas rencontré de problèmes de communication ou de feed-back avec ses membres.

\section{Appendice 1}

\section{Organigramme}

Santé-Planification Familiale

Directrice de Project

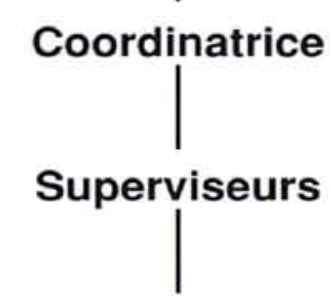

Agents de Terrain<smiles>C[AsH3-]</smiles>

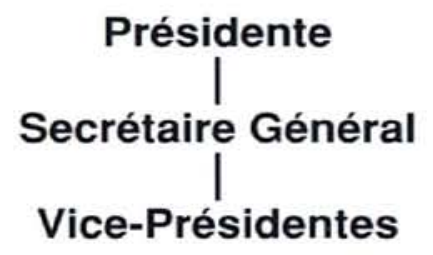

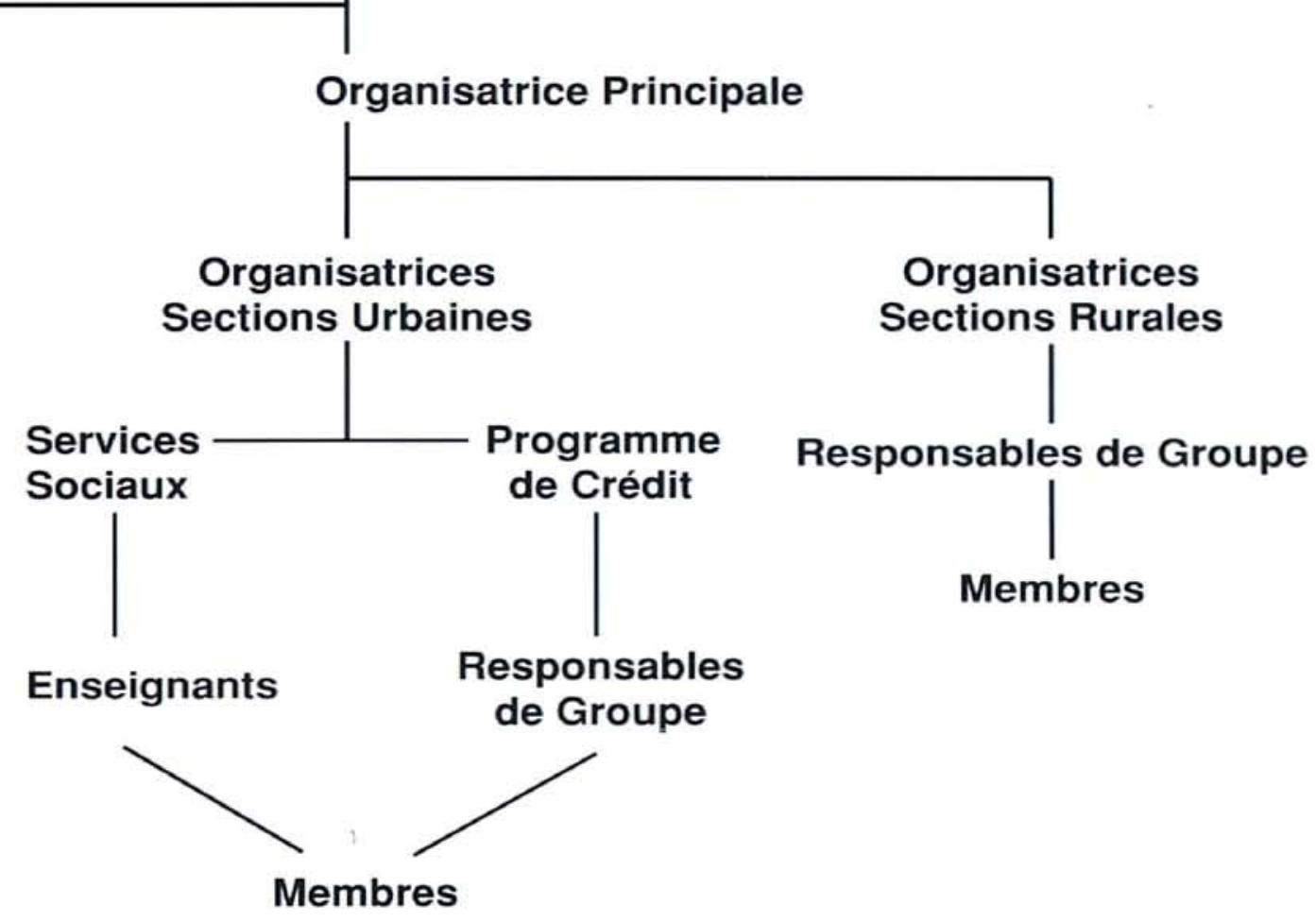


Membres Généraux-Conseil Général. Les membres généraux, c'est-à-dire, les membres titulaires ou les membres associés, constituent le Conseil Général du Forum. Ils se rencontrent régulièrement (au moins une fois par mois) dans leurs groupes de quartier respectifs, de temps à autre, avec des membres appartenant à d'autres groupes, et, une fois par an, à l'occasion de réunions publiques importantes ou pour d'autres fonctions. Au cours des réunions individuelles ou locales, les responsables locaux et les organisatrices régionales essayent d'insuffler la discipline et l'idéologie du Forum aux participantes. Pour citer une organisatrice régionale "Nous organisons des réunions pour expliquer les statuts et les règlements du Forum aux membres et pour les inciter à assister régulièrement aux réunions." Dans le même temps, les membres généraux ont la possibilité d'aborder les problèmes et les questions auxquels ils sont confrontés et pour lesquels ils voudraient qu'une solution soit trouvée.

Responsables locales-Corps exécutif. Les responsables locales (actuellement au nombre de 320) constituent le corps exécutif du Forum. Elles assistent à des réunions de coordination mensuelles au siège du Forum au cours desquelles les problèmes des différents groupes et membres sont passés en revue. Elles sont ensuite tenus de faire un compte rendu et d'informer les membres généraux de toute décision ayant été prise les concernant. Elles exercent également un certain nombre d'activités clés au sein du Forum: évaluation des demandes de prêts des différents membres, et collecte et dépôt à la banque des versements mensuels des différents individus. Les responsables de groupe travaillent à titre bénévole mais, en contrepartie, elles ont droit à des prêts plus importants que les membres généraux.

Organisatrices régionales-Agents de terrain. En raison de l'accroissement considérable du nombre de ses adhérantes, le Forum a décidé de promouvoir des responsables de groupe motivées et compétentes à des postes d'organisatrices locales pour superviser plusieurs groupes dans leur région. Leur travail consiste à rencontrer, au moins une fois par semaine, les responsables des groupes dont elles ont la charge, à se rendre au bureau du FFA au minimun deux fois par semaine, et à superviser les enseignants travaillant dans les crèches ou les écoles de nuit. Elles évaluent également les demandes de prêt soumises par les responsables de groupe, expliquent les statuts et les règlements du Forum à ces dernières, et leur aident en cas de défaut de paiement de l'un des membres.

Responsable des Prêts, Organisatrice Principale-Personnel Administratif. Lorsque le Forum pris la décision d'ouvrir sa propre Société de Crédit Mutuel, on recruta une organisatrice principale et une responsable des prêts (à nouveau parmi les membres généraux). La responsable des prêts a la décision finale sur toutes les demandes de prêts et les défauts de paiement. Elle aide également à traiter les demandes de prêts. L'Organisatrice principale supervise et assiste les organisatrices régionales dans leur travail.

Fonctionnaires-Commission Permanente. Les fonctionnaires permanents sont les suivants: trois organisatrices régionales, un secrétaire-général, deux vice-présidentes, et une présidente. C'est à eux que revient la décision finale sur toutes les questions administratives et financières. Les deux vice-présidentes et le secrétaire général sont également des organisatrices régionales dans leurs quartiers respectifs. De temps à autre, on les envoie en mission pour s'assurer du bon déroulement des opérations lorsque le Forum ouvre de nouvelles branches.

Fondatrice-Présidente. En raison de ses qualifications et de son expérience, la présidente exerce des responsabilités distinctes et séparées, notamment: (a) négociations et liaison avec les banques, les bailleurs de fonds, le gouvernement et autres organisations; (b) établissement du calendrier de remboursement des prêts avec les banques; (c) administration; (d) sollicitation de fonds; et (e) réunions occasionnelles avec les groupes et visites sur le terrain. 
Mise en page: Ann Leonard

Typographie: Village Type and Graphics

Photos: Marty Chen

Imprimeur: Graphic Impressions, Inc.

Traduction: Pia Heitz

Nous attendons vos remarques, commentaires et idées de projets à publier dans les prochains numéros de SEEDS. Si vous souhaitez recevoir des exemplaires supplémentaires de ce numéro ou faire partie de nos abonnés, n'hésitez pas à nous écrire. Envoyez votre courrier à:

Ann Leonard, Conseillère à la Rédaction SEEDS

P.O. Box Box 3923

Grand Central Station

New York, New York 10163 U.S.A. 


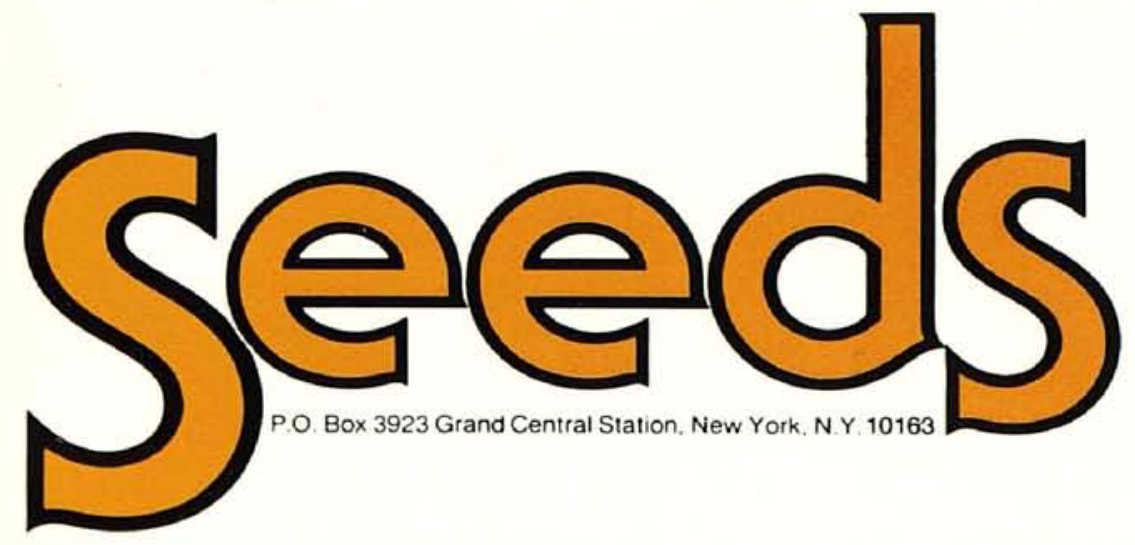

